\title{
Genetic algorithm with a crossover elitist preservation mechanism for protein-ligand docking
}

\author{
Boxin Guan $\mathbb{B}^{0}$, Changsheng Zhang* and Jiaxu Ning
}

\begin{abstract}
Protein-ligand docking plays an important role in computer-aided pharmaceutical development. Protein-ligand docking can be defined as a search algorithm with a scoring function, whose aim is to determine the conformation of the ligand and the receptor with the lowest energy. Hence, to improve an efficient algorithm has become a very significant challenge. In this paper, a novel search algorithm based on crossover elitist preservation mechanism (CEP) for solving protein-ligand docking problems is proposed. The proposed algorithm, namely genetic algorithm with crossover elitist preservation (CEPGA), employ the CEP to keep the elite individuals of the last generation and make the crossover more efficient and robust. The performance of CEPGA is tested on sixteen molecular docking complexes from RCSB protein data bank. In comparison with GA, LGA and SODOCK in the aspects of lowest energy and highest accuracy, the results of which indicate that the CEPGA is a reliable and successful method for protein-ligand docking problems.
\end{abstract}

Keywords: Protein-ligand docking, Pharmaceutical development, Genetic algorithm, AutoDock, Crossover elitist preservation

\section{Introduction}

Protein-ligand docking is one of the most important methods in structure-based pharmaceutical development (Brooijmans and Kuntz 2003; Huang and Zou 2010; Jug et al. 2015; Moitessier et al. 2008; Zhao et al. 2014, 2016), and it is also an important approach for large-scale virtual screening. With the development of X-ray technology, the three-dimensional structure of docked conformations has been obtained so that protein-ligand docking has more practical significance. Through the establishment of protein-ligand docking model, and researching the interaction the receptor and the ligand, to discover and design a more effective, more ideal drugs. The process of molecular docking is to search conformations of the proteins and the ligands with lowest energy. The ligands are placed at the active site of the protein receptors, and reasonable orientations and conformations are sought to

\footnotetext{
*Correspondence: zhangchangsheng@ise.neu.edu.cn

Key Laboratory of Medical Image Computing of Ministry of Education, Northeastern University, Shenyang 110819, People's Republic of China
}

match the shape and interaction of ligands and receptors. The active binding site refers to a specific small region in the receptors, which is composed of a small number of amino acid residues on the side chain. The optimized target energy value of molecular docking is obtained by calculating the interaction between the ligands and the binding region of the receptors.

Scoring function (Hu et al. 2004; Huey et al. 2006; Jain 2006; Muryshev et al. 2003) and search algorithm (Blum et al. 2011; López-Camacho et al. 2015) are two important parts in the process of protein-ligand docking. The scoring function which is a force field to evaluate the energy of the docking conformation is helpful to explore the binding model receptors and ligands. Reasonable scoring function not only can correctly assess the docking results, but it also can distinguish the difference between the results of different docking (Bharatham et al. 2014; Li et al. 2015).

The search algorithm is to find out the optimal binding mode between small ligand and its receptor protein around binding site. Some algorithms have been shown 
to be very effective for solving the protein-ligand docking problem, and some researchers have improved the power of these docking methods. For example, simulated annealing (SA) (Goodsell and Olson 1990), Genetic algorithm (GA) (Cao and Li 2004; Jones et al. 1997; Thomsen 2003), Lamarckian genetic algorithm (LGA) (Fuhrmann et al. 2010), SODOCK (Chen et al. 2007; Jason et al. 2008; $\mathrm{Ng}$ et al. 2015), and artificial bee colony algorithm (ABC) (Uehara et al. 2015). However, to develop an efficient and reliable search algorithm is still a challenge for docking problem.

The parents of the elitist individual in original genetic algorithm are not retained, which lead to good genes of the parents do not continue produce to excellent individual through crossover operation. In the article, a new evolutionary algorithm, namely genetic algorithm with crossover elitist preservation (CEPGA), is presented to overcome the shortcoming. The introduction of the crossover elitist preservation (CEP) mechanism can improve the speed of operation and ensure that the optimal solution is not abandoned. The next generation is better for the competition of the elitist parents and their offspring. Moreover, a local search which can select a optimal solution in the near space of the current solution is incorporated into the GA.

AutoDock is a protein-ligand docking software developed by Morris et al. of Scripps Research Institute in the United States. AutoDock (Kitchen et al. 2004; Morris et al. 2009) is a free and open source docking software, and it is also the most widely used automated docking program. The software first produces the grid of the binding site, and then uses the search algorithm to find the best combination of the receptor and the ligand, and finally evaluates the conformation by means of the scoring function. AutoDock 4.2.6 is used as an experimental environment in this paper. The semi-empirical free energy force field that is based on a overall thermodynamic model which can convert intramolecular energy into binding and predictive free energy in AutoDock 4.2.6 is used as a scoring function in the experiments of the paper. To study the capability of the presented method, genetic algorithm crossover elitist preservation mechanism (CEPGA), it has been tested on a set of different protein-ligand complexes from RCSB protein data bank (PDB) (http://www.rcsb.org/pdb) (Berman et al. 2002) and compared to GA, LGA, SODOCK, and ABC.

\section{Materials and methods}

\section{Standard genetic algorithm}

Genetic algorithm (GA) is a method by simulating Darwin's theory of natural evolution to search for the optimal solution. Genetic algorithm starts from a population contains potential solutions of a specific problem. Each encoding corresponds to a solution for the problem, and it called a individual or chromosome. Then with the help of selection, crossover, and mutation produces a new population. This process results in that the population evolves from generation to generation to get more and better approximate solutions according to the principle of survival of the fittest. The best individual which is decoded in the last population can be used as an optimal solution. On the basis of the ability of the individual to adapt to the environment, selection decides the survival or the elimination of the individual. The selection operation enables the individuals with higher fitness which is evaluated using the scoring function to be preserved with greater probability, so that the population converges to the global optimum at the fastest speed. Sort selection is a ranking of all individuals according to their fitness values and determines the probability of individuals being selected, it is used in GA for protein-ligand docking. The process in which individuals randomly pair up, exchange part of their chromosomes at a probability, and form new individuals is called crossover. One point crossover, an intersection is randomly selected and two individuals swap at the front or back of the point to produce a new individual, is adopted as crossover operator of GA for protein-ligand docking. The so-called mutation, which is a number of accidental factors, causes the genes in individuals are randomly transformed at a certain probability and produces new individuals. For the protein-ligand docking problem, GA is real code, so real mutation is used as mutation operator.

\section{Genetic algorithm with crossover elitist preservation mechanism}

The crossover of genetic algorithm, first of all, two relative paired individuals are determined based on specific principles. Then, they exchange some genes in a specific way to form two new individuals. The purpose of crossover is to keep the good genes of the parent generation and generate a lot of new individuals. However, the pairing of the individuals is random in the parent generation, and the randomness plays an ineffective role in the global search. The excellent individuals of the previous generation have not been retained due to the randomness, and the individuals may not be as good as the previous generation. Accordingly, a novel crossover strategy is introduced.

In the method, $\mathrm{X}_{0}, \mathrm{X}_{\text {father }}$ and $\mathrm{X}_{\text {mother }}$ are introduced. $\mathrm{X}_{0}$ represents elitist individual, $\mathrm{X}_{\text {father }}$ represents the father of elitist individual, and $X_{\text {mother }}$ represents the mother of elitist individual. When the current solution is better than any other solutions before, the current solution is defined as $\mathrm{X}_{0}$ and $\mathrm{X}_{\text {father }}$ and $\mathrm{X}_{\text {mother }}$ of $\mathrm{X}_{0}$ are preserved. The saved value of the parents are used for 
the next crossover operation. With the development of the algorithm, using good values of $\mathrm{X}_{\text {father }}$ and $\mathrm{X}_{\text {mother }}$ instead of other values for crossover, the search algorithm are gradually efficient. The new method is called crossover elitist preservation mechanism and abbreviated as CEP.

Example: suppose CEPGA randomly generates six individuals, 1a, 1b, 1c, 1d, 1e and 1f, respectively, in the first generation. In Fig. 1 (1), six new individuals are produced by crossover operator of GA in the second generation, such as $1 \mathrm{a}$ and $1 \mathrm{~b}$ cross to generate $2 \mathrm{a}$. If the individual $2 \mathrm{a}$ is the current optimal solution, the parents of the elitist individual, $1 \mathrm{a}$ and $1 \mathrm{~b}$, are preserved. Because the genes of the parents of the elitist individual are excellent, they may be more likely to reproduce elitist individuals. Then the preserved individuals, $1 \mathrm{a}$ and $1 \mathrm{~b}$, replace the individuals, $2 \mathrm{a}, 2 \mathrm{~b}, 2 \mathrm{c}, 2 \mathrm{~d}, 2 \mathrm{e}$, and $2 \mathrm{f}$, in the second generation. $2 \mathrm{a}$ as the elitist individual can not be replaced. Two random individuals of the remaining five individuals are selected in the second generation, such as $2 b$ and $2 c$, and then replace them with 1a and 1b. In Fig. 1 (2), 2b and 2c are replaced by saved individuals $1 \mathrm{a}$ and $1 \mathrm{~b}$, so the next generation is $2 a, 1 a, 1 b, 2 d, 2 e, 2 f$.

By using the CEP, the parents of the elitist individual and the population of current generation are combined to make the gene quality of the population better, ensures that the genes of good individuals are not discarded during evolution, and maintain that the genes of the best individuals in the population can pass on to the next generation. For protein-ligand docking, the number of elitist individuals is $\theta$ *the number of population, where $\theta$ is a particular adjustable number (the range is 0.01-0.1). Hence, the number of the parents of elitist individuals is

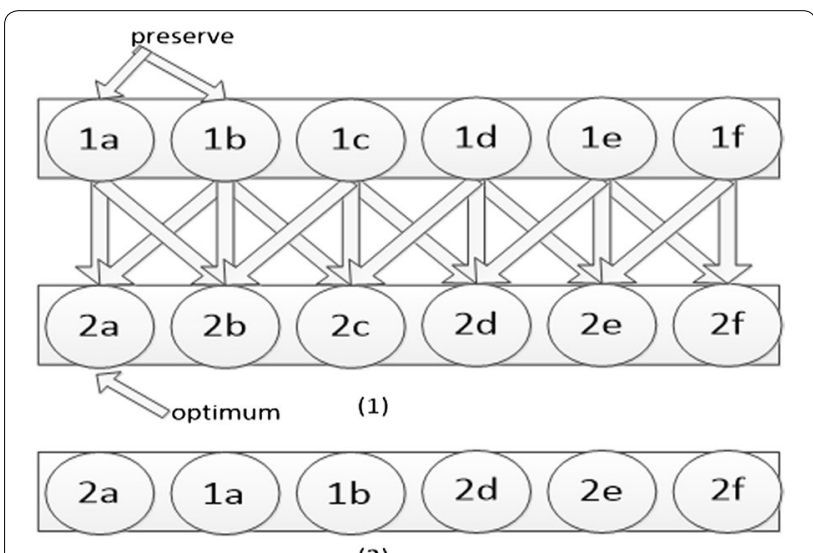

(2)

Fig. 1 Diagram of CEP. (1) The individuals of the previous generation pair and cross to generate the individuals of the next generation. The optimum individual 2a serves as an elitist individual, and its parents are preserved. (2) The adjusted individuals of the next generation after CEP
$2 \theta^{*}$ the number of population. The parents of the elitist individuals are preserved, and they replace individuals of current generation except the elitist individuals.

Local search is an algorithm chooses an optimal solution in the near solution space of the current solution, until it reaches a local optimal solution. The basic idea of local search algorithm: search direction is carried out along the direction of the solution of the target. If a solution is not a local optimum, the local search can get a optimal solution in its near space. In the search process, the locally strong search algorithm always selects the neighborhood of the current solutions. The local search is also added to the novel algorithm (CEPGA) in order to improve the efficiency.

The pseudo-code and the block diagram of CEPGA is showed in Table 1 and Fig. 2, respectively. CEPGA begins with a random initialized population. Then, the next population is reproduced after crossover, CEP (steps 04-11), mutation and selection. From the second generation, elitist individuals with good genes are reproduced, and the parents of these elitist individuals are preserved. The preserved individuals of parent generation and the individuals of sub-generation are combined to form a new parent population. The introduction of the crossover elitist preservation strategy can increase the sampling space and the competition among individuals. It is easier to get a better solution through the competition among the elitist individuals in the new formed generation. This process continues until a specific termination condition is reached. The above steps ensure that the best genes are not destroyed and the algorithm evolves toward the direction of the optimal solution.

\section{Results}

To value the impact of the presented algorithm, the performance found by CEPGA with GA, LGA, SODOCK and $\mathrm{ABC}$ is compared. The semi-empirical free energy force field described above is used in all experiments in this paper. In order to maintain the diversity of the protein-ligand X-ray structures, theses instances should have a wide span of the number of rotatable bonds in ligands. Sixteen protein-ligand X-ray structures $(\mathrm{Hu}$ et al. 2004) with $0-15$ rotatable bonds in ligands are chosen from RCSB protein data bank (Berman et al. 2002) (http://www.rcsb.org/pdb) to compare the capability of the docking techniques.

\section{(1) 3ptb beta-trypsin/ben (benzamidine)}

Beta-trypsin is a kind of protease, which is extracted from the pancreas of cattle, sheep and pigs. Benzamidine is an inhibitor, and it is often used to prevent proteolytic degradation of proteins. 
Table 1 Pseudo-code of CEPGA

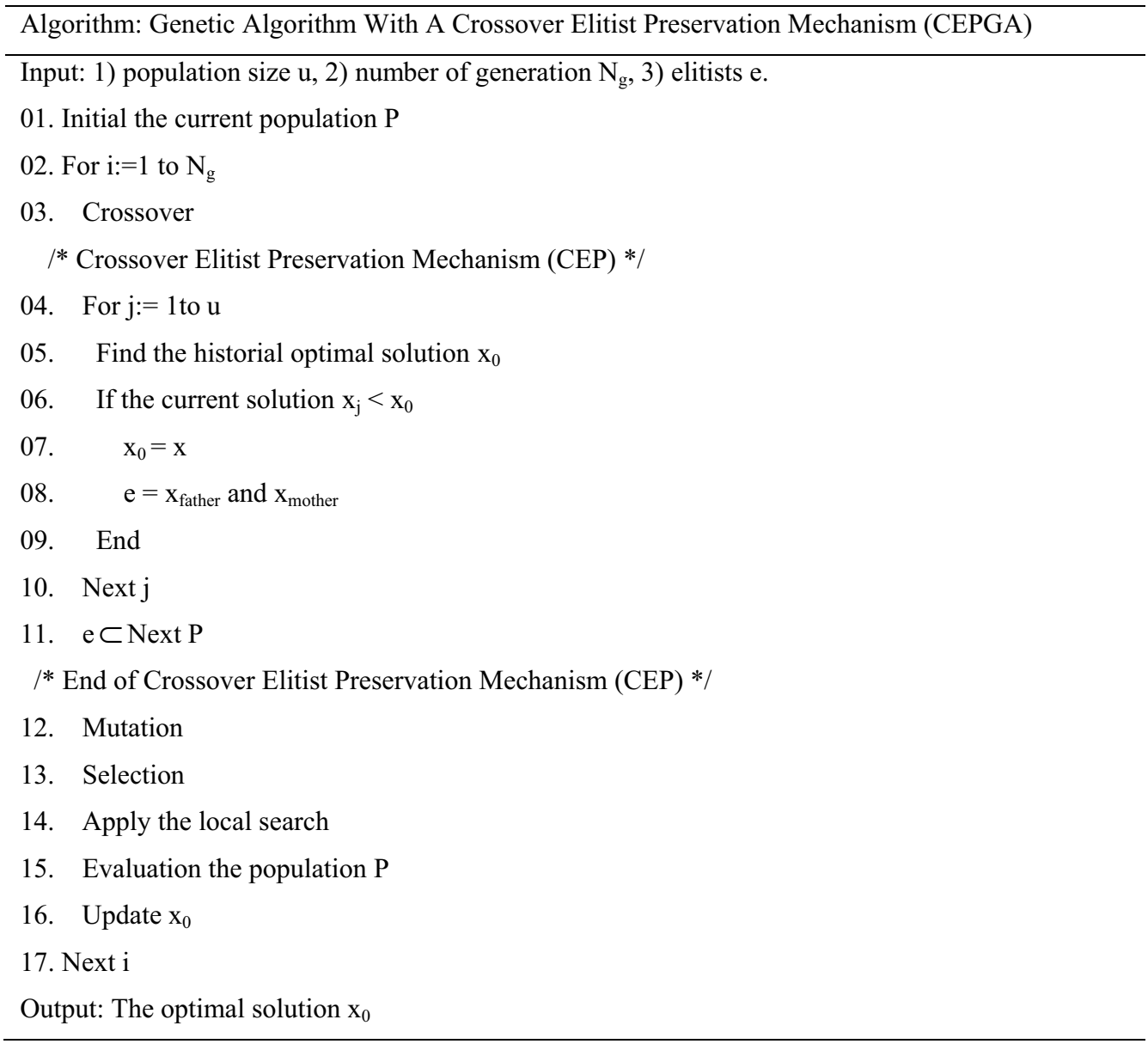

(2) 1aha alpha-momorcharin/ade (adenine)

Alpha-momorcharin is extracted from the seeds of Momordica charantia. Adenine is a substance in the body.

\section{(3) 3hvt HIV-1 reverse transcriptase/nvp}

HIV-1 reverse transcriptase is the three phosphate enzyme that synthesizes complementary DNA. Nvp is a potent, non-nucleoside reverse transcriptase inhibitor.

\section{(4) 1 phg cytochrome P450-cam/hem (protoporphyrin IX)} Cytochrome P450-cam is a superfamily of heme-thiolate proteins, it is involved in the metabolism of endogenous and exogenous substances. Protoporphyrin IX is purple brown crystalline powder, soluble in methanol, insoluble in water, chloroform, ether and acetone.

\section{(5) $2 \mathrm{mcp}$ McPC-603/pc (phosphocholine)}

McPC-603 is a phosphocholine-binding mouse myeloma protein. Phosphocholine is an intermediate in the synthesis of phosphatidylcholine in tissues.

\section{(6) 1 stp streptavidin/btn (biotin)}

Biotin, also known as vitamin $\mathrm{H}$ or coenzyme $\mathrm{R}$, is a water-soluble B-vitamin. Streptavidin/Biotin is one of the most tightly binding noncovalent complexes. 窗体顶端

Streptavidin is a kind of protein that gained from streptomyces, and it has a similar biological characteristic with affinity. Biotin is one of the B vitamins, and it is essential for the normal metabolism of fats and proteins.

\section{(7) 6rnt ribonuclease $\mathrm{T} 1 / \mathrm{ca}$ (calcium ion)}

Ribonuclease $\mathrm{T} 1$ is a endonuclease that removes the non hybridized RNA region in the DNA-RNA hybrid. Calcium ion is an indispensable ion in the physiological activities of the body.

\section{(8) 4dfr dihydrofolate reductase/mtx (methotrexate)} 窗体顶端

Dihydrofolate reductase is an enzyme that has been used as a drug-target in the building of anti-cancer and other processes. Methotrexate is an substance that has a 


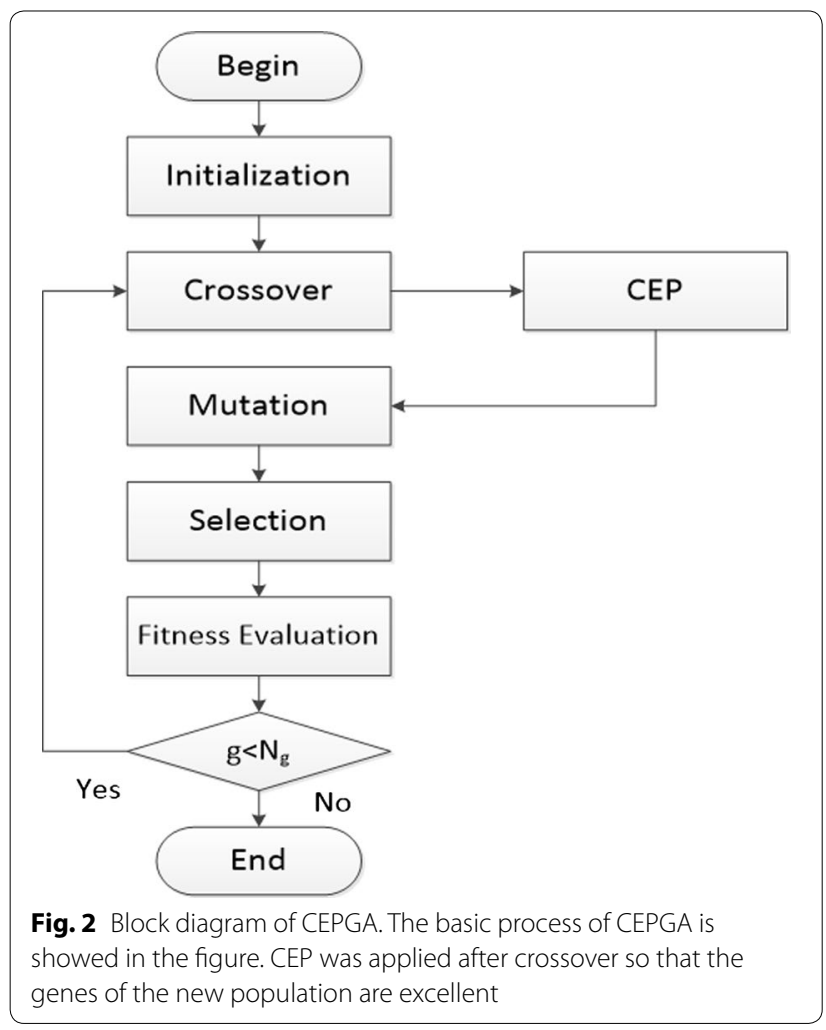

strong immunosuppressive effect, it can prevent division and proliferation of immune cells.

\section{(9) 1ett thrombin/4qq}

Thrombin is a white to gray amorphous material, and it is generally freeze-dried powder. 4qq is a non-polymer inhibitor.

\section{(10) 1 hri human rhinovirus/s57}

Human rhinovirus is a kind of rhinovirus and the main cause of the common cold in humans. S57 is a kind of imidazole.

\section{(11) $1 \mathrm{hvr}$ protease/xk2}

Protease is an enzyme that catalyzes protein catabolism, and it can be find in plants, animals, and so on. Xk2 is an small molecule inhibitor that can block or reduce the rate of chemical reaction.

\section{(12) $4 \mathrm{hmg}$ hemagglutinin/sia (sialic acid)}

Hemagglutinin is a substance that results in red blood cells to coagulate. Sialic acids are acidic monosaccharides which are produced at terminal sugars chains.

\section{(13) 1cdg cyclodextrin glycosyl transferase/mol (maltose)} 窗体顶端
Cyclodextrin glycosyltransferase is a bacterial enzyme which has the ability to generate cyclodextrins. Maltose is a substance formed from malt and starch, and it is used as a nutrient and a culture medium.

\section{(14) 1 htf HIV-1 protease/g26}

HIV-1 protease is an enzyme that separates newly synthesized polyproteins to their component peptides. G26 is a non-polymer inhibitor. G26 is a kind of amide which is a highly reactive and easily oxidizable perssad.

\section{(15) 1glq glutathione S-transferase/gtb (S-(P-nitrobenzyl) glutathione)}

Glutathione S-transferase is a group of enzymes related to the detoxification function of the liver. S-(P-nitrobenzyl)Glutathione is an important synthesis of glutathione precursor.

\section{(16) $1 \mathrm{tmn}$ thermolysin/nas (2-naphthalenesulfonic acid)}

Thermolysin is a biological substance, and it is characterized by the hydrolysis of hydrophobic amino acids at a faster rate. 2-naphthalenesulfonic acid is white crystal or powder, soluble in water, insoluble in alcohol, and it can be used in organic synthesis.

The AutoDock's PDBQTs of the protein and the ligand are prepared firstly. The PDBQT of the protein is obtained using the following steps: (1) read protein. (2) remove water molecules. (3) add hydrogen. The ligand follow the lowing procedure to get the PDBQT format: (1) read ligand. (2) detect root. (3) choose torsion. (4) set number of torsion.

It is necessary to make sure that the parameters of different search algorithms are equally set up. Therefore, in the three GAs, the population is 50 , the number of generations is 27,000 , and the energy evaluations is $1.5 \times 10^{6}$ in a docking. In this way, the dockings are terminated by reaching the maximum number of generations. In the SODOCK, the number of particles and immediate neighbors is 50 and 5 , respectively; while the maximal number of function evaluations is $1.5 \times 10^{6}$. And in the $A B C$, the number of the population is 50 , and the maximum number of cycles is $1.5 \times 10^{6}$.

Each method is run ten times independent for each protein-ligand docking problem. Table 2 lists the protein-ligand complex names (PDB), the ligand names, the number ligand torsions, the lowest energies and the smallest RMSDs for all 16 test proteins. RMSD is the root mean square deviation between the docking results and the crystal complex, and it is the most important index to evaluate the docking accuracy. It is acceptable if the RMSD is less than $2.0 \AA$, otherwise the docking is invalid. Through the results table, It is concluded that the CEPGA finds 13 lowest energy of thirteen in the 16 molecular 
Table 2 Lowest energy and smallest RMSD results of five compared algorithms

\begin{tabular}{|c|c|c|c|c|c|c|c|c|c|c|c|}
\hline \multirow[b]{2}{*}{ PDB } & \multirow[b]{2}{*}{ Ligand (torsions) } & \multicolumn{2}{|l|}{ CEPGA } & \multicolumn{2}{|l|}{ LGA } & \multicolumn{2}{|l|}{ GA } & \multicolumn{2}{|c|}{ SODOCK } & \multicolumn{2}{|l|}{$A B C$} \\
\hline & & Energy & RMSD & Energy & RMSD & Energy & RMSD & Energy & RMSD & Energy & RMSD \\
\hline $3 p t b$ & ben $(0)$ & -11.72 & 1.90 & -11.46 & 1.92 & -10.31 & 1.66 & -11.57 & 2.00 & -10.90 & 1.97 \\
\hline 1aha & ade (1) & -15.32 & 0.89 & -16.10 & 0.45 & -15.16 & 1.28 & -14.95 & 1.44 & -13.90 & 1.80 \\
\hline 3hvt & $\operatorname{nvp}(2)$ & -17.90 & 0.30 & -17.22 & 0.33 & -15.73 & 0.43 & -16.78 & 0.58 & -15.60 & 0.55 \\
\hline $1 \mathrm{phg}$ & hem (3) & -9.32 & 0.64 & -8.56 & 0.80 & -7.46 & 1.20 & -8.95 & 1.54 & -7.95 & 1.67 \\
\hline $2 m c p$ & $\mathrm{pc}(4)$ & -9.10 & 1.20 & -8.22 & 1.33 & -7.76 & 1.46 & -7.72 & 1.42 & -7.80 & 1.54 \\
\hline $1 \mathrm{stp}$ & btn (5) & -13.57 & 0.90 & -13.37 & 1.65 & -11.03 & 1.84 & -13.52 & 1.00 & -13.17 & 1.68 \\
\hline $6 \mathrm{rnt}$ & $\mathrm{ca}(6)$ & -9.32 & 0.58 & -9.13 & 0.70 & -8.58 & 0.69 & -9.12 & 1.95 & -8.90 & 1.55 \\
\hline $4 \mathrm{dfr}$ & $\operatorname{mtx}(7)$ & -12.12 & 1.90 & -11.44 & 1.23 & -10.01 & 0.95 & -11.34 & 1.60 & -10.21 & 1.97 \\
\hline 1ett & 4qq (8) & -14.21 & 1.29 & -13.89 & 1.38 & -11.42 & 1.62 & -12.06 & 1.56 & -12.70 & 1.70 \\
\hline $1 \mathrm{hri}$ & s57 (9) & -10.89 & 1.38 & -10.21 & 1.87 & -9.67 & 1.80 & -10.31 & 1.68 & -10.13 & 1.67 \\
\hline $1 \mathrm{hvr}$ & xk2 (10) & -31.06 & 0.64 & -30.85 & 0.62 & -21.95 & 1.68 & -29.29 & 0.68 & -28.64 & 0.85 \\
\hline $4 \mathrm{hmg}$ & sia (11) & -10.32 & 1.89 & -10.09 & 1.70 & -8.44 & 1.69 & -10.08 & 1.36 & -9.80 & 1.54 \\
\hline 1cdg & mol (12) & -8.70 & 1.45 & -8.22 & 1.94 & -7.32 & 1.69 & -8.45 & 1.80 & -7.13 & 1.12 \\
\hline $1 \mathrm{htf}$ & g26 (13) & -21.48 & 1.27 & -20.69 & 1.33 & -18.86 & 1.46 & -21.79 & 1.42 & -19.17 & 1.96 \\
\hline 1glq & gtb (14) & -9.46 & 1.38 & -9.27 & 1.87 & -7.97 & 1.87 & -8.83 & 1.90 & -9.13 & 1.60 \\
\hline $1 \mathrm{tmn}$ & nas (15) & -10.29 & 0.85 & -10.11 & 1.20 & -9.68 & 1.11 & -10.62 & 1.95 & -9.37 & 0.60 \\
\hline
\end{tabular}

docking complexes. The smallest RMSD found by each of the five search algorithms is 9,2, 2, 1, and 2 using CEPGA, LGA, GA, SODOCK, and ABC respectively.

The convergence diagrams are illustrated in Fig. 3. The experiment records the optimal energy as the vertical axis and the number of energy evaluations when the optimal energy value is evaluated as the horizontal axis. The convergence curve and the convergence period of the algorithm are observed, which provides a reference for the performance evaluation. Figure 4 shows box plots between five compared algorithms in different PDB. The energy values of each PDB are arranged from large to small, and the upper edge, the upper quartile, median, the median, the lower four quantile, and the lower edge are calculated, respectively. Under the confidence level of 0.05 , we adopt hypothesis test (Knowles et al. 2006) to demonstrate whether CEPGA can be applied to all protein-ligand docking problem in Table 3. When comparing algorithm 1 with algorithm 2, the algorithm 1 is superior to the algorithm 2 if the p value is less than 0.05 .

\section{Discussion}

Drug molecular design plays a decisive role in the development of drugs. Protein-ligand docking is the major method of computer aided drug design (Guedes et al. 2014; Huang and Zou 2010), which takes advantage of the combination of drug chemistry and computer technology to improve the efficiency of drug development (Zhao et al. 2008, 2011). The aim of protein-ligand docking is to find the best ligand conformation of a ligand against a protein target with the lowest energy (Bohlooli et al. 2017). many researchers have made great efforts to improve the power of the protein-ligand docking methods, such as simulated annealing (SA), genetic algorithm (GA) (Jones et al. 1997), Lamarckian genetic algorithm (LGA) (Fuhrmann et al. 2010), SODOCK (Chen et al. 2007), and artificial bee colony (ABC) (Uehara et al. 2015). However, the quality of the solutions that the existing algorithms obtain is insufficient. This paper illustrates a novel and robust optimization algorithm (CEPGA) for solving the protein-ligand docking problems with an aim to overcome the above-mentioned drawback.

An efficient docking method consists of two connected goals, which are the fitness accuracy (energy based) and the pose accuracy (root mean square deviation (RMSD) based) (Guo et al. 2014; Hu et al. 2004). For the fitness accuracy, the lower energy is associated with the greater binding activity which can also give rise to better drug efficiency. RMSD is utilized to determine whether two docked conformations are similar enough to be categorized into the same cluster. A docked conformation with a smaller RMSD is considered as a more accurate solution to the docking problem. Compared CEPGA with GA, LGA, SODOCK, and ABC (Castro-Alvarez et al. 2017; Feinstein and Brylinski 2015), Table 2 show that CEPGA has the best performance in the search for the lowest energy and the smallest RMSD of molecular docking conformations.

We also evaluate the performance of CEPGA in other aspects including convergence analysis, data distribution, 


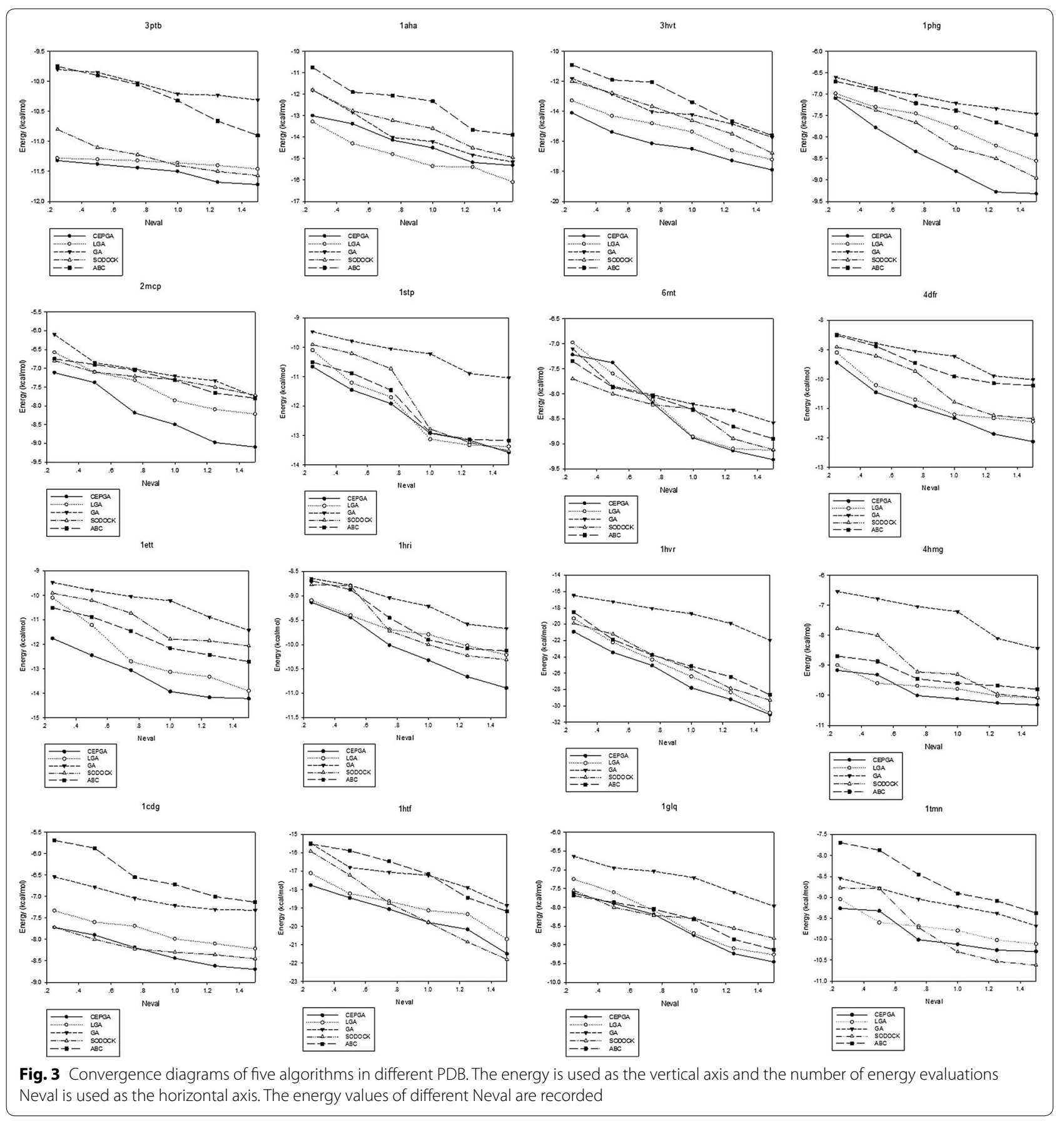

and hypothesis test (Knowles et al. 2006) in comparison with GA, LGA, SODOCK, and ABC (Castro-Alvarez et al. 2017; Feinstein and Brylinski 2015). The convergence diagrams (Fig. 3) indicate that CEPGA is superior to other methods in terms of convergence rate and solution quality, and these figures also show that CEPGA can prevent premature convergence. For data distribution, as seen in box plots (Fig. 4), the medians of CEPGA are the lowest and its data are the most concentrated. This can demonstrate that CEPGA is a stable algorithm for protein-ligand docking. Hypothesis tests are showed in Table 3, and it can be obviously seen that CEPGA is better than other algorithms according the $\mathrm{p}$ value in the tables.

In conclusion, the paper presents the CEPGA which combines genetic algorithms, crossover elitist 

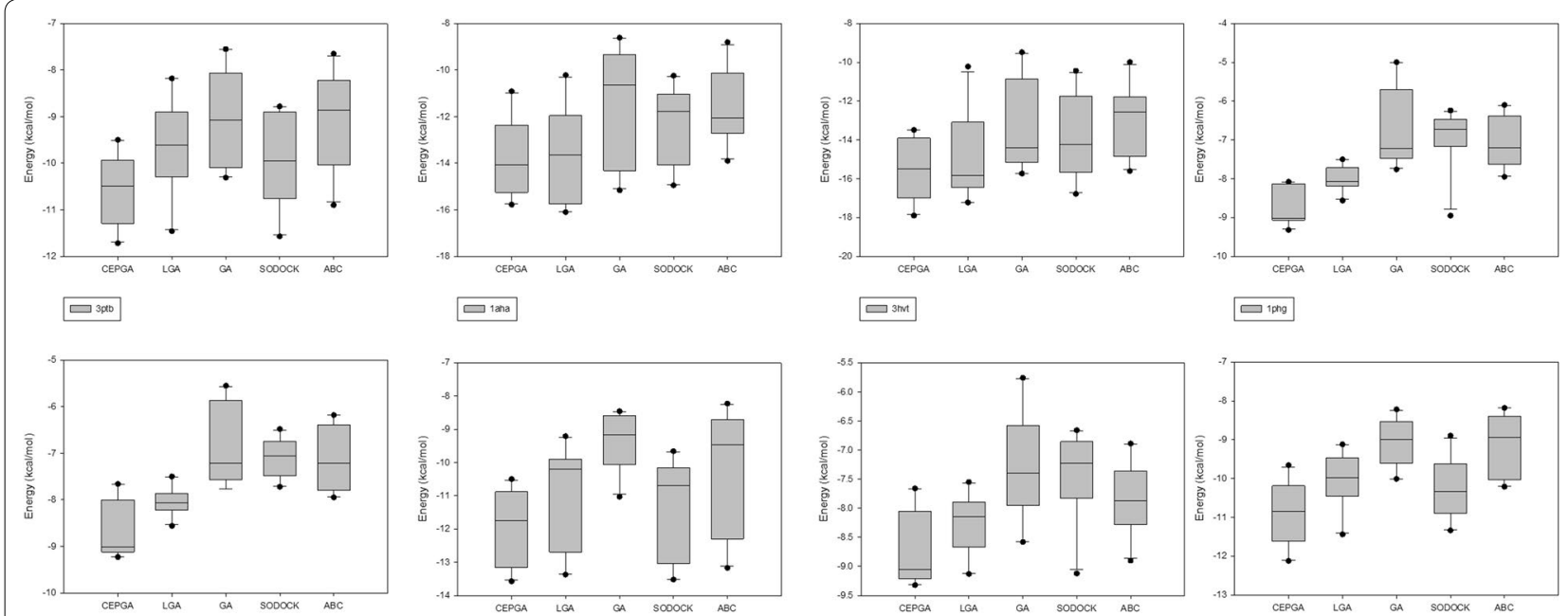

\section{口住a}
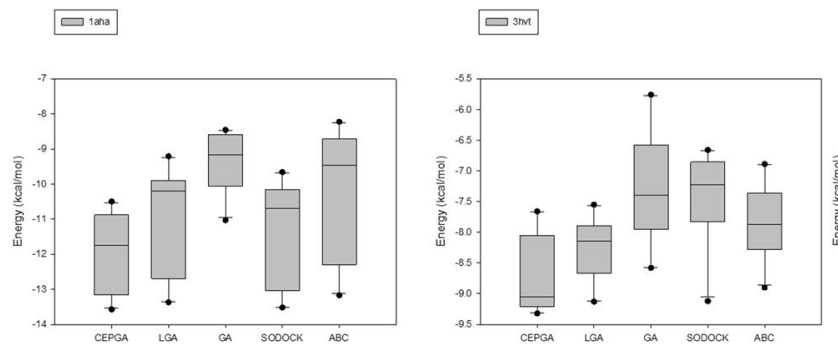

$\square^{1009}$

$$
\square^{2 m c p}
$$

$\square^{\text {ss }}$
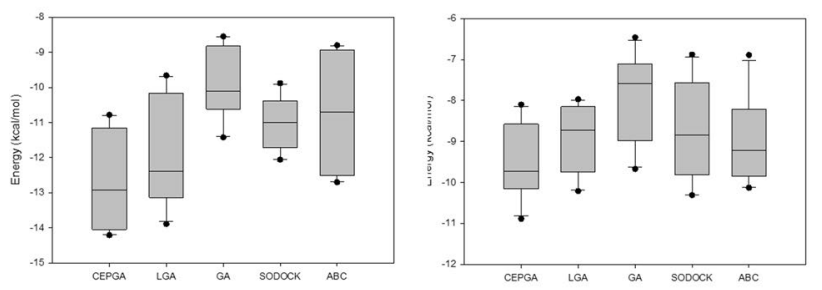

$$
\square \mathrm{cmt}
$$
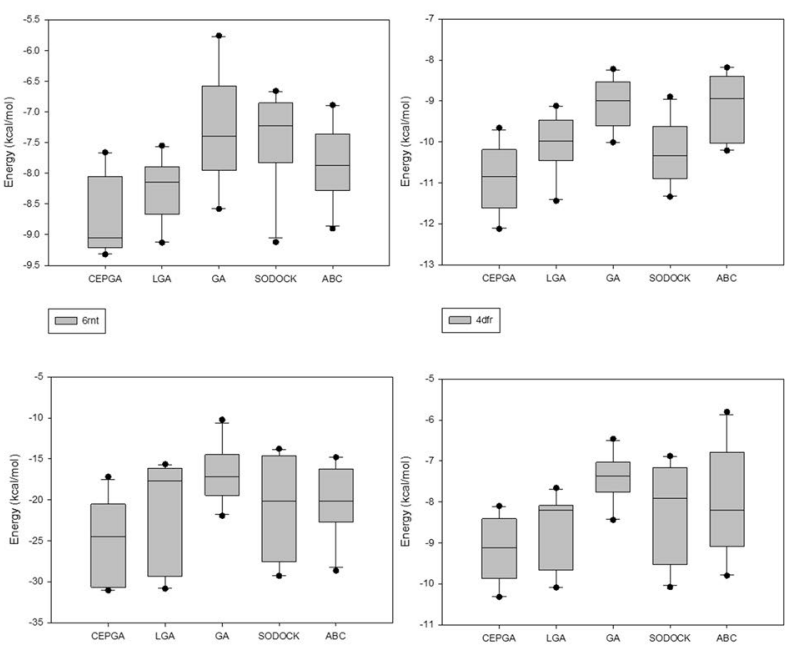

$\square_{\text {tatr }}$

$\square^{\mathrm{mit}}$
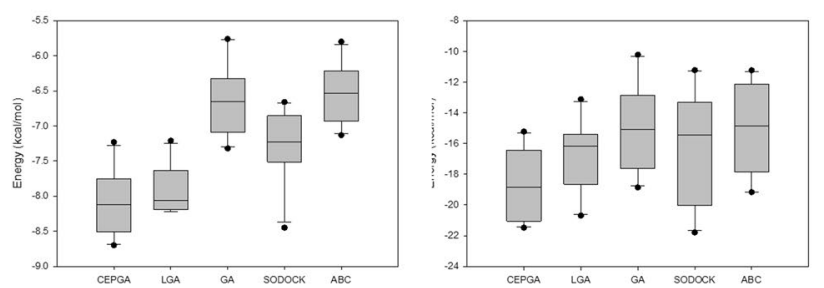

$\square$ int

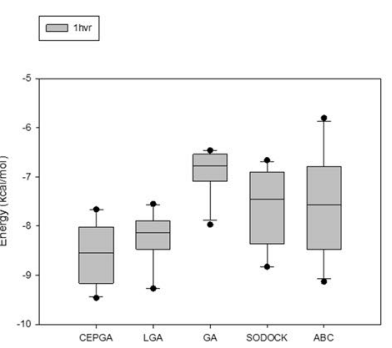

$\square^{190}$

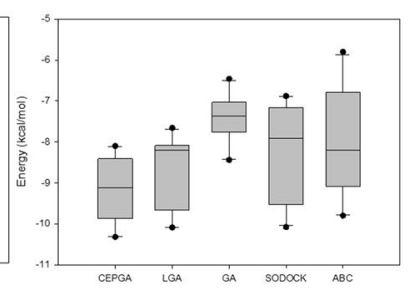

$\square$ amg

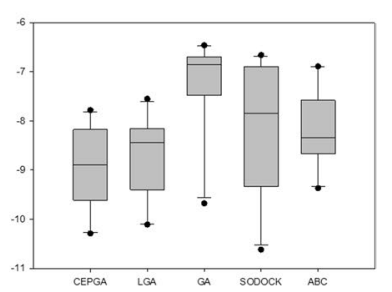

$\square^{\text {thn }}$

Fig. 4 Box plots of five algorithms in different PDB. The energy is used as the vertical axis and the five compared algorithms are used as the horizontal axis. The energy values of the upper edge, the upper quartile, the median, median, the lower four quantile, and the lower edge are recorded, respectively

preservation (CEP), and local search method to extends the power of the GA_based algorithm for molecular docking problems. By using the CEP mechanism, the search algorithm not only can retain elitists to improve the efficiency of crossover, but also can get better energy value and RMSD. The five search methods, CEPGA, LGA, GA, SODOCK, and ABC are tested by experiments above. The results indicate that CEPGA has superior 
Table 3 Hypothesis test result

\begin{tabular}{|c|c|c|c|c|c|c|c|c|c|c|c|}
\hline & CEPGA & LGA & GA & SODOCK & $A B C$ & & CEPGA & LGA & GA & SODOCK & $A B C$ \\
\hline $3 p t b$ & & & & & & 1ets & & & & & \\
\hline CEPGA & - & 0.012 & 0.004 & 0.028 & 0.006 & CEPGA & - & 0.025 & 0.009 & 0.015 & 0.018 \\
\hline LGA & 0.988 & - & 0.008 & 0.563 & 0.225 & LGA & 0.975 & - & 0.018 & 0.063 & 0.127 \\
\hline GA & 0.996 & 0.992 & - & 0.995 & 0.688 & GA & 0.991 & 0.982 & - & 0.595 & 0.688 \\
\hline SODOCK & 0.972 & 0.437 & 0.005 & - & 0.100 & SODOCK & 0.985 & 0.937 & 0.405 & - & 0.504 \\
\hline$A B C$ & 0.994 & 0.775 & 0.312 & 0.900 & - & $A B C$ & 0.982 & 0.873 & 0.312 & 0.496 & - \\
\hline 1aha & & & & & & 1hri & & & & & \\
\hline CEPGA & - & 0.519 & 0.402 & 0.124 & 0.105 & CEPGA & - & 0.038 & 0.002 & 0.040 & 0.015 \\
\hline LGA & 0.481 & - & 0.036 & 0.017 & 0.004 & LGA & 0.962 & - & 0.014 & 0.723 & 0.151 \\
\hline $\mathrm{GA}$ & 0.598 & 0.964 & - & 0.342 & 0.260 & GA & 0.998 & 0.986 & - & 0.982 & 0.637 \\
\hline SODOCK & 0.976 & 0.983 & 0.658 & - & 0.470 & SODOCK & 0.960 & 0.277 & 0.012 & - & 0.020 \\
\hline$A B C$ & 0.895 & 0.996 & 0.740 & 0.530 & - & $A B C$ & 0.985 & 0.849 & 0.363 & 0.980 & - \\
\hline 3hvt & & & & & & $1 \mathrm{hvr}$ & & & & & \\
\hline CEPGA & - & 0.035 & 0.007 & 0.023 & 0.013 & CEPGA & - & 0.043 & 0.005 & 0.011 & 0.009 \\
\hline LGA & 0.965 & - & 0.205 & 0.324 & 0.150 & LGA & 0.957 & - & 0.038 & 0.177 & 0.044 \\
\hline $\mathrm{GA}$ & 0.993 & 0.795 & - & 0.778 & 0.437 & GA & 0.995 & 0.962 & - & 0.942 & 0.565 \\
\hline SODOCK & 0.977 & 0.676 & 0.222 & - & 0.215 & SODOCK & 0.989 & 0.823 & 0.058 & - & 0.168 \\
\hline$A B C$ & 0.987 & 0.850 & 0.463 & 0.585 & - & $A B C$ & 0.991 & 0.956 & 0.435 & 0.832 & - \\
\hline 1phg & & & & & & $4 \mathrm{hmg}$ & & & & & \\
\hline CEPGA & - & 0.036 & 0.008 & 0.041 & 0.017 & CEPGA & - & 0.020 & 0.005 & 0.017 & 0.010 \\
\hline LGA & 0.964 & - & 0.016 & 0.624 & 0.450 & LGA & 0.980 & - & 0.008 & 0.417 & 0.214 \\
\hline $\mathrm{GA}$ & 0.992 & 0.984 & - & 0.988 & 0.537 & $\mathrm{GA}$ & 0.995 & 0.992 & - & 0.988 & 0.900 \\
\hline SODOCK & 0.959 & 0.376 & 0.012 & - & 0.215 & SODOCK & 0.983 & 0.583 & 0.012 & - & 0.240 \\
\hline$A B C$ & 0.983 & 0.550 & 0.463 & 0.785 & - & $\mathrm{ABC}$ & 0.990 & 0.786 & 0.100 & 0.760 & - \\
\hline $2 \mathrm{mcp}$ & & & & & & $1 \mathrm{cdg}$ & & & & & \\
\hline CEPGA & - & 0.013 & 0.004 & 0.002 & 0.003 & CEPGA & - & 0.017 & 0.006 & 0.044 & 0.005 \\
\hline LGA & 0.987 & - & 0.203 & 0.182 & 0.224 & LGA & 0.983 & - & 0.117 & 0.763 & 0.105 \\
\hline GA & 0.996 & 0.797 & - & 0.492 & 0.610 & GA & 0.994 & 0.883 & - & 0.985 & 0.408 \\
\hline SODOCK & 0.997 & 0.818 & 0.508 & - & 0.640 & SODOCK & 0.956 & 0.237 & 0.015 & - & 0.012 \\
\hline$A B C$ & 0.995 & 0.776 & 0.390 & 0.360 & - & $A B C$ & 0.995 & 0.895 & 0.592 & 0.988 & - \\
\hline $1 \mathrm{stp}$ & & & & & & $1 \mathrm{htf}$ & & & & & \\
\hline CEPGA & - & 0.034 & 0.007 & 0.042 & 0.013 & CEPGA & - & 0.148 & 0.023 & 0.640 & 0.015 \\
\hline LGA & 0.964 & - & 0.009 & 0.624 & 0.450 & LGA & 0.852 & - & 0.027 & 0.883 & 0.151 \\
\hline GA & 0.993 & 0.991 & - & 0.992 & 0.487 & GA & 0.977 & 0.973 & - & 0.987 & 0.637 \\
\hline SODOCK & 0.958 & 0.376 & 0.008 & - & 0.215 & SODOCK & 0.360 & 0.127 & 0.013 & - & 0.017 \\
\hline$A B C$ & 0.987 & 0.550 & 0.513 & 0.785 & - & $A B C$ & 0.985 & 0.849 & 0.363 & 0.983 & - \\
\hline 6rnt & & & & & & 1glq & & & & & \\
\hline CEPGA & - & 0.035 & 0.008 & 0.029 & 0.010 & CEPGA & - & 0.045 & 0.009 & 0.049 & 0.042 \\
\hline LGA & 0.965 & - & 0.018 & 0.368 & 0.127 & LGA & 0.955 & - & 0.018 & 0.163 & 0.227 \\
\hline GA & 0.992 & 0.982 & - & 0.695 & 0.588 & GA & 0.991 & 0.982 & - & 0.695 & 0.788 \\
\hline SODOCK & 0.971 & 0.632 & 0.305 & - & 0.404 & SODOCK & 0.951 & 0.837 & 0.305 & - & 0.704 \\
\hline$A B C$ & 0.990 & 0.873 & 0.412 & 0.496 & - & $A B C$ & 0.958 & 0.773 & 0.212 & 0.296 & - \\
\hline $4 \mathrm{dfr}$ & & & & & & $1 \mathrm{tmn}$ & & & & & \\
\hline CEPGA & - & 0.015 & 0.005 & 0.011 & 0.008 & CEPGA & - & 0.317 & 0.008 & 0.744 & 0.095 \\
\hline LGA & 0.985 & - & 0.009 & 0.337 & 0.115 & LGA & 0.683 & - & 0.217 & 0.763 & 0.105 \\
\hline GA & 0.995 & 0.991 & - & 0.986 & 0.685 & GA & 0.992 & 0.783 & - & 0.905 & 0.408 \\
\hline SODOCK & 0.989 & 0.663 & 0.014 & - & 0.142 & SODOCK & 0.256 & 0.237 & 0.005 & - & 0.012 \\
\hline$A B C$ & 0.992 & 0.885 & 0.315 & 0.858 & - & $A B C$ & 0.995 & 0.895 & 0.592 & 0.988 & - \\
\hline
\end{tabular}

Table 3 continued 
ability to the other four search algorithms in terms of robustness and efficiency. This suggests that CEPGA can enhance the applicability of AutoDock to docking problems.

\author{
Abbreviations \\ CEP: crossover elitist preservation; CEPGA: genetic algorithm with crossover \\ elitist preservation; SA: simulated annealing; GA: genetic algorithm; LGA: \\ Lamarckian genetic algorithm; ABC: artificial bee colony algorithm; RMSD: \\ root-mean-square deviation.
}

\section{Authors' contributions}

BG planned and carried out the experiments, analyzed the data and wrote the manuscript; CZ reviewed the manuscript; JN participated in the data analysis. All authors read and approved the final manuscript.

\section{Authors' information}

Boxin Guan is a Ph.D. candidate in computer science, Northeastern University, China. His major research interests include evolutionary computation and bioinformatics. Changsheng Zhang is an associate professor in the School of Computer Science and Engineering, Northeastern University, China. His major research interests include data mining, evolutionary computation, and bioinformatics. Jiaxu Ning is a Ph.D. candidate in computer science, Northeastern University, China. Her major research interests include machine learning and bioinformatics.

\section{Acknowledgements}

We are grateful to Prof. Yuhai Zhao for excellent technical assistance.

\section{Competing interests}

The authors declare that they have no competing interests.

\section{Availability of data and materials}

All relevant data are presented in the manuscript.

\section{Consent for publication}

Not applicable.

\section{Funding}

This work was supported by the National Natural Science Foundation Program of China $(61572116,61572117,61502089,61772124)$, the Fundamental Research Funds for the Central Universities (N150402002) and the National key Technology R\&D Program of the Ministry of Science and Technology (2015BAH09F02).

\section{Publisher's Note}

Springer Nature remains neutral with regard to jurisdictional claims in published maps and institutional affiliations.

Received: 30 May 2017 Accepted: 5 September 2017

Published online: 13 September 2017

\section{References}

Berman HM, Battistuz T, Bhat TN, Bluhm WF, Bourne PE, Burkhardt K, Feng Z, Gilliland GL, lype L, Jain S, Fagan P, Marvin J, Padilla D, Ravichandran V, Schneider B, Thanki N, Weissig H, Westbrook JD, Zardecki C (2002) The protein data bank. Acta Crystallogr D Biol Crystallogr 58:899-907

Bharatham N, Bharatham K, Shelat AA, Bashford D (2014) Ligand binding more prediction by docking: $\mathrm{mdm} 2 / \mathrm{mdmx}$ inhibitors as a case study. J Chem Inf Model 54(2):648-659

Blum C, Puchinger J, Raidl GR, Roli A (2011) Hybrid mataheuristics in combinatorial optimization: a survey. Appl Soft Comput 11(6):4135-4151

Bohlooli F, Sepehri S, Razzaghi-AsI N (2017) Response surface methodology in drug design: a case study on docking analysis of a potent antifungal fluconazole. Comput Biol Chem 67:158-173
Brooijmans N, Kuntz ID (2003) Molecular recognition and docking alogirthms. Annu Rev Biophys Biomol Struct 32(32):335-373

Cao TC, Li TH (2004) A combination of numeric genetic algorithm and tabu search can be applied to molecular docking. Comput Biol Chem 28(4):303-312

Castro-Alvarez A, Costa AM, Vilarrasa J (2017) The performance of several docking programs at reproducing protein-macrolide-like crystal structures. Molecules 22(1):136

Chen HM, Liu BF, Huang HL, Hwang SF, Ho SY (2007) SODOCK: swarm optimization for highly flexible protein-ligand docking. J Comput Chem 28(2):612-623

Feinstein WP, Brylinski M (2015) Calculating an optimal box size for ligand docking and virtual screening against experimental and predicted binding pockets. J Cheminform 7:18

Fuhrmann J, Rurainsk A, Lenhof HP, Neumann D (2010) A new Lamarckian genetic algorithm for flexible ligang-receptor docking. J Comput Chem 31(9):1911-1918

Goodsell DS, Olson AJ (1990) Automated docking of substrates to proteins by simulated annealing. Proteins Struct Funct Genet 8(3):195-202

Guedes IA, de Magalhães CS, Dardenne LE (2014) Receptor-ligand molecular docking. Biophys Rev 6(1):75-87

Guo LY, Yan ZQ, Zheng XL, Hu L, Yang YL, Wang J (2014) A comparison of various optimization algorithms of protein-ligand docking programs by fitness accuracy. J Mol Model 20:2251

Hu X, Balaz S, Shelver WH (2004) A practical approach to docking of zinc metalloproteinase inhibitors. J Mol Graph Model 22(4):293-307

Huang SY, Zou XQ (2010) Advances and challenges in protein-ligand docking. Int J Mol Sci 11(8):3016-3034

Huey R, Morris GM, Olson AJ, Goodsell DS (2006) Software news and update a semiempirical free energy force field with charge-based desolvation. J Comput Chem 10:1145-1152

Jain AN (2006) Scoring functions for protein-ligand docking. Curr Protein Pept Sci 7(5):407-420

Jason S, Merkle D, Middendorf M (2008) Molecular docking with multi-objective particle swarm optimization. Appl Soft Comput 8(1):666-675

Jones G, Willett P, Glen RC, Leach AR, Taylor R (1997) Development and validation of a genetic algorithm for flexible docking. J Mol Biol 267(3):727-748

Jug G, Anderluh M, Tomašič T (2015) Comparative evaluation of several docking tools for docking small molecule ligands to DC-SIGN. J Mol Model 21(6):164-178

Kitchen DB, Decomez H, Furr JR, Bajorath J (2004) Docking and scoring in virtual screening for drug discovery: methods and applications. Nat Rev Drug Discov 3:935-949

Knowles J, Thiele L, Zitzler E (2006) A tutorial on the performance assessment of stochastic multiobjective optimizers. Computer Engineering and Networks Laboratory (TIK), ETH Zurich

Li ZF, Gu JF, Zhuan HY, Kang L, Zhao XY, Guo G (2015) Adaptive molecular docking method baesd on information entropy genetic algorithm. Appl Soft Comput 26:299-302

López-Camacho E, Godoy MJ, Garcỉa-Nieto J, Nebro AJ, Aldana-Montes JF (2015) Solving molecular flexible docking problems with mataheuristics: a comparative study. Appl Soft Comput 28(6):379-393

Moitessier N, Englebienne P, Lee D, Lawandi J, Gorbeil CR (2008) Towards the development of universal, fast and highly accurate docking/scoring methods: a long way to go. Br J Pharmacol 153(1):7-26

Morris GM, Huey R, Lindstrom W, Sanner MF, Belew RK, Goodsell DS, Olson AJ (2009) AutoDock4 and AutoDockTools4: automated docking with selective receptor flexibility. J Comput Chem 30(16):2785-2791

Muryshev AE, Tarasov DN, Butygin AV, Butygina OV, Aleksandrov AB, Nikitin SM (2003) A novel scoring function for molecular docking. J Comput Aided Mol Des 17(9):597-605

Ng MC, Fong S, Siu SW (2015) PSOVina: the hybrid particle swarm optimization algorithm for protein-ligand docking. J Bioinform Comput Biol 13(3):1541007

Thomsen R (2003) Flexible ligand docking using evolutionary algorithms: investigating the effects of variation operators and local search hybrids. Biosystems 72:57-73

Uehara S, Fujimoto KJ, Tanaka S (2015) Protein-ligand docking using fitness learning-based artificial bee colony with proximity stimuli. Phys Chem Chem Phys 17(25):16412-16417 
Zhao YH, Jeffrey XY, Wang GR (2008) Maximal Subspace Coregulated Gene Clustering. IEEE Trans Knowl Data Eng 20(1):83-98

Zhao YH, Wang GR, Li Y, Wang ZH (2011) Finding novel diagnostic gene patterns based on interesting non-redundant contrast sequence rules. ICDM. p 972-981

Zhao YH, Wang GR, Zhang X, Yu JX, Wang ZH (2014) Learning phenotype structure using sequence model. IEEE Trans Knowl Data Eng 26(3):667-681
Zhao YH, Wang GR, Yin Y (2016) Improving ELM-based microarray data classification by diversified sequence features selection. Neural Comput Appl 27(1):155-166

\section{Submit your manuscript to a SpringerOpen ${ }^{\odot}$ journal and benefit from:}

- Convenient online submission

- Rigorous peer review

- Open access: articles freely available online

High visibility within the field

- Retaining the copyright to your article

Submit your next manuscript at $\boldsymbol{\nabla}$ springeropen.com 